\title{
Plankton: Population Dynamics Models
}

\section{Francois Carlotti}

Aix-Marseille Université, Université de Toulon, CNRS/INSU, IRD, MIO, UM 110

Mediterranean Institute of Oceanography

13288, Marseille, Cedex 09, France 


\section{GLOSSARY}

SPM : Structured population models

IBM : Individual-based models

ROMS: Regional Ocean Modeling System

KtW model: Killing-the-Winner model

Darwin model: high-resolution ocean and ecosystem model with many plankton species ECOPATH: free ecological/ecosystem modeling software (www.ecopath.org) 


\section{KEY WORDS:}

Plankton,

Population Dynamics Models,

Structured population models,

Individual-based models,

Matrix population models,

McKendrick-von Foerster equation,

Bioenergetic processes,

Eulerian models,

Grid-based models,

Lagrangian biological models 


\begin{abstract}
:
Population models of plankton species are used to describe and predict changes in abundance, distribution, and production of populations in their habitat conditions. The chapter presents succinctly the different mathematical formulations used for planktonic population models which belong to two types of approaches: structured population models and individual-based models. The recent development in building population models concern more mechanistic descriptions of organism biological properties (physiology, behavior and life-history traits) improving its modeled representation, and the coupling with 3D hydrodynamical models to understand retention-dispersion processes and connectivity between metapopopulations, and in fine population response and adaptation to environmental variability.
\end{abstract}




\section{INTRODUCTION}

The general purpose of population models of plankton species is to describe and eventually predict the changes in abundance, distribution, and production of targeted populations in their habitat conditions, i.e. under forcing of the abiotic environment, food conditions and predation. Computerbased approaches in plankton ecology were introduced during the 1970 with the application of population models to investigate large-scale population phenomena by the use of mathematical models.

Today, virtually every major scientific research project of population ecology has a modeling component. Population models are built for four main objectives, eventually for some practical purposes of management: (1) to estimate the survival of individuals and the persistence of populations in their habitat, and to look at the factors and processes which regulate their variability, (2) to estimate the flow of energy and matter through a given population, and its role in trophic food webs; (3) to study different aspects of behavioral ecology, and (4) to evaluate how genetic heterogeneity among populations impact the population structure of species and to identify transformations in biological traits that provide the adaptation to specific habitat condition.

The study of interactions between populations and successions of population are also topics related to population models. The field of biological modeling has diversified, and at present, complex mathematical approaches like neural networks, genetic algorithms and dynamical optimization start to be used thanks to the availability of supercomputers. However, the use of models in marine research should always be accompanied by extensive field data and laboratory experiments, either for initialization, verification or falsification, or continuous updating.

In recent years, plankton ecology has significantly grown from new data types with increased resolution of data acquisition with video, optical and acoustic plankton sensors on various platforms in relation with the physical environment, as well as from more comprehensive studies of organism physiology, behavior and life-history traits. New approaches to model marine population have 
emerged (i.e. trait-based and size-based modeling) using more mechanistic descriptions of organism biology improving its modeled representation (Lindeman et al 2017).

\section{APPROACH FOR MODELLING PLANKTON POPULATION}

\section{Population structure and units}

A population is defined as a group of living organisms all of one species restricted in a given area and with limited exchanges of individuals from other populations. Thus, gene flows between quasi isolated populations are low in comparison with the significant gene flows within each population. The first step in building a population model is to identify state variables (components of the population) and to describe the interactions between these state variables and external variables of the system and among the components themselves. The components of a population can be: 1) the entire population (1 component); 2) groups of individuals identified by a certain state: developmental stages, weight or size classes, age classes (fixed numbers of components), 3) all individuals or cohorts of individuals of similar history (varying numbers of components).

The usual unit in population dynamics models is the number of individuals per volume of water, but the population biomass can also be used (in $\mathrm{g}$ biomass or $\mathrm{C}$ or $\mathrm{N}$ ). When all individuals or groups of individuals are represented, the individual weight can be considered as a state variable.

The forcing variables influencing the population dynamics are biological factors, mainly trophic nutriments and predators, and physical factors, mainly temperature, light, advection and diffusion.

\section{Individual and demographic processes}

Population models usually include four major processes: individual growth, development, reproduction and mortality. Growth is computed by the rate of individual body weight change. 
Development is represented by the change of states (phases in phytoplankton and protozoan cell division, developmental stages in zooplankton) through which each individual progresses to reach maturity. Reproduction is represented by the production of new individuals. Mortality induces loss of individuals, and can be divided in two components: natural physiological mortality (due for instance to starvation) and predation. Combination of these four processes permits one to simulate (1) increase in terms of number of individuals in the population, (2) the body growth of these individuals, and (3) by combination of the two previous simulated values, the increase in total biomass of the population (which is usually termed "population growth").

\section{Plankton Characteristics}

The marine plankton community comprises a very large range of organisms from virus and prokaryotes to eukaryotes, uni- and multicellular, invertebrates and vertebrates, covering a wide size range and presenting a great variety of life cycles. Therefore the initial conceptual framework to build a population model structure requires identification of scales of the main biological characteristics of the studied species (Figure 1). Thus, essential information to build models of plankton organisms are: (1) the individual life duration (few hours for bacteria, one to few days for phytoplankton and unicellular animals, several weeks to years for zooplankton and ichthyoplankton organisms) and stage durations for those with complex life-cycles, (2) the range of change in size or weight between the beginning and the end of a life cycle (which is limited to a doubling in size in unicellular organisms, whereas the change in size and weight for metazoans are of several orders of magnitude and through different life stages), (3) the behavior and the swimming capacity on vertical and horizontal axis; and (4) the number of individuals produced by a mother individual (two individuals up to thousands of individuals). The observation time step has to be defined to adequately follow the time scale of the chosen variables, and thus should be lower than the duration of the shortest phases. 


\section{Production rate Species Life cycle Behaviour}

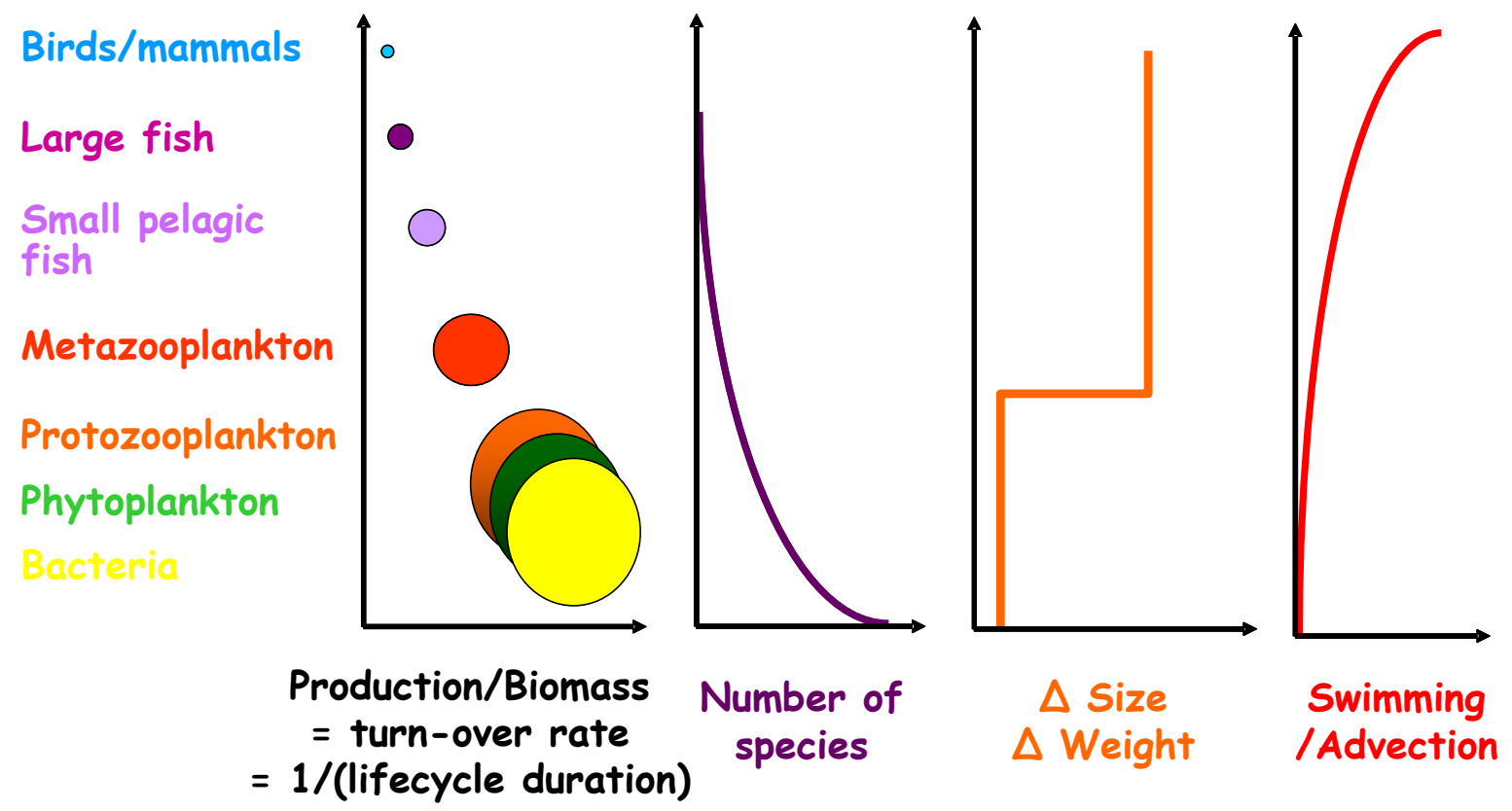

Figure 1 Schematic representation of key differences in biological rates, species numbers, and size/weight growth during the life cycle, and swimming behaviour between the different trophic levels in marine ecosystems. Modified from Carlotti F and Poggiale J.C. 2010. Towards methodological approaches to implement the zooplankton component in "end to end" food-web models. Progress in Oceanography 84: 20-38.

\section{Plankton population models}

Modeling plankton dynamics coupled to physical models and nutrient dynamics allows advancing our understanding of how pelagic marine ecosystems respond to climate variability. Therefore, the most modeled component in marine planktonic ecosystems is phytoplankton production. Most of the earliest phytoplankton models simulated the growth of phytoplankton as a whole, using only the process of photosynthesis. Nowadays, biogeochemical models consider different functional groups of phytoplanktonic populations, and a few models are dedicated to phytoplankton population growth dynamics at the species level including functional traits. Existing models of other unicellular 
plankton organisms (bacterioplankton, microzooplankton) usually treat them as a single unit, except for a few models simulating microbial cell cycles. On the contrary, mesozooplankton organisms, including the planktonic larval stages of benthic species (meroplankton) and fish with complex life cycles are extensively modeled at the population level.

Several types of modeling approaches can represent population demographics, including physiological traits and intra-population variability. The population can be considered as bulk or can be divided into discrete developmental stages (or size classes) with the model computing the inflow and outflow of individuals for each stage. Those approaches are suitable for simulating intrapopulation variability using one state variable. For multiple state variables (e.g. age, size/weight and reserve pool), matrix, individual-based or agent-based techniques can be used.

\section{DYNAMICS OF SINGLE SPECIES}

\section{Populations models described by the total density}

When a population is observed at timescales much larger than the individual life span, and on a large number of generations, models with one variable (the total number of individuals or the total biomass in that population) are the simplest. These models postulate that the rate of change of the population number, $\mathrm{N}$, is proportional to $\mathrm{N}$ (eqn [1], where $\mathrm{r}$ is the difference between birth and death rates).

$$
\frac{\mathrm{dN}}{\mathrm{dt}}=\mathrm{rN}
$$

The logistic equation ([2]) represents limitation due to the resources or space (see Figure 2).

$$
\frac{\mathrm{dN}}{\mathrm{dt}}=\mathrm{rN}\left(1-\frac{\mathrm{N}}{\mathrm{K}}\right)
$$

where $\mathrm{K}$ is the carrying capacity. 
Population growth of bacteria, phytoplankton, or microzooplankton can be simulated adequately by the logistic equation. With addition of a time delay term into the logistic equation, oscillations of the population can be represented.

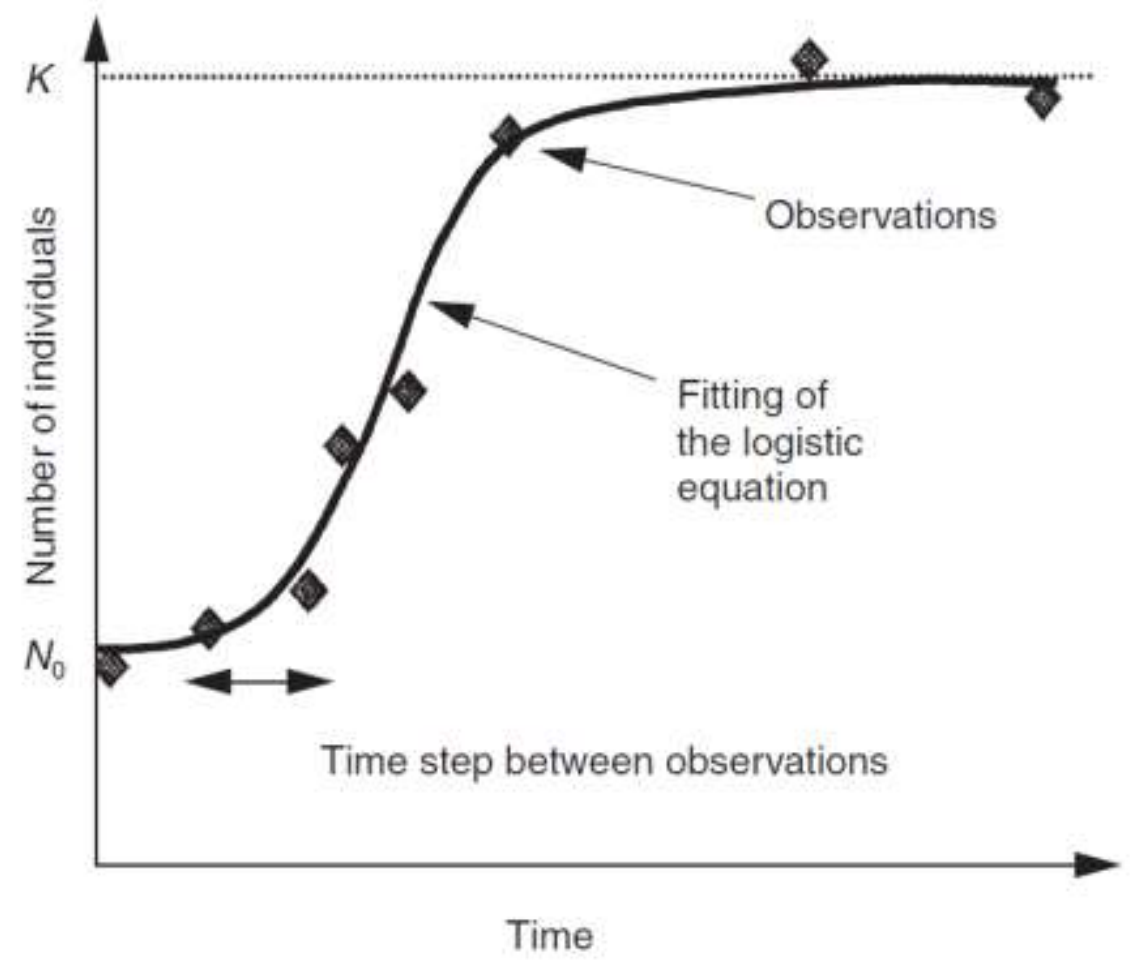

Figure 2 The growth of a plankton population with density regulation and its fitting by the logistic equation.

\section{Population models of organisms with description of the life cycle}

If observations of a population are made with a time step shorter than the life cycle duration (Figure 3), the population development pattern is a succession of periods with decreasing abundance of individuals due to mortality, and increasing abundance due to recruitment of new individuals in periods of reproduction (cell division or egg production). Recruitment is defined as the input flux of individuals in a given state (stage, size class, etc.). 


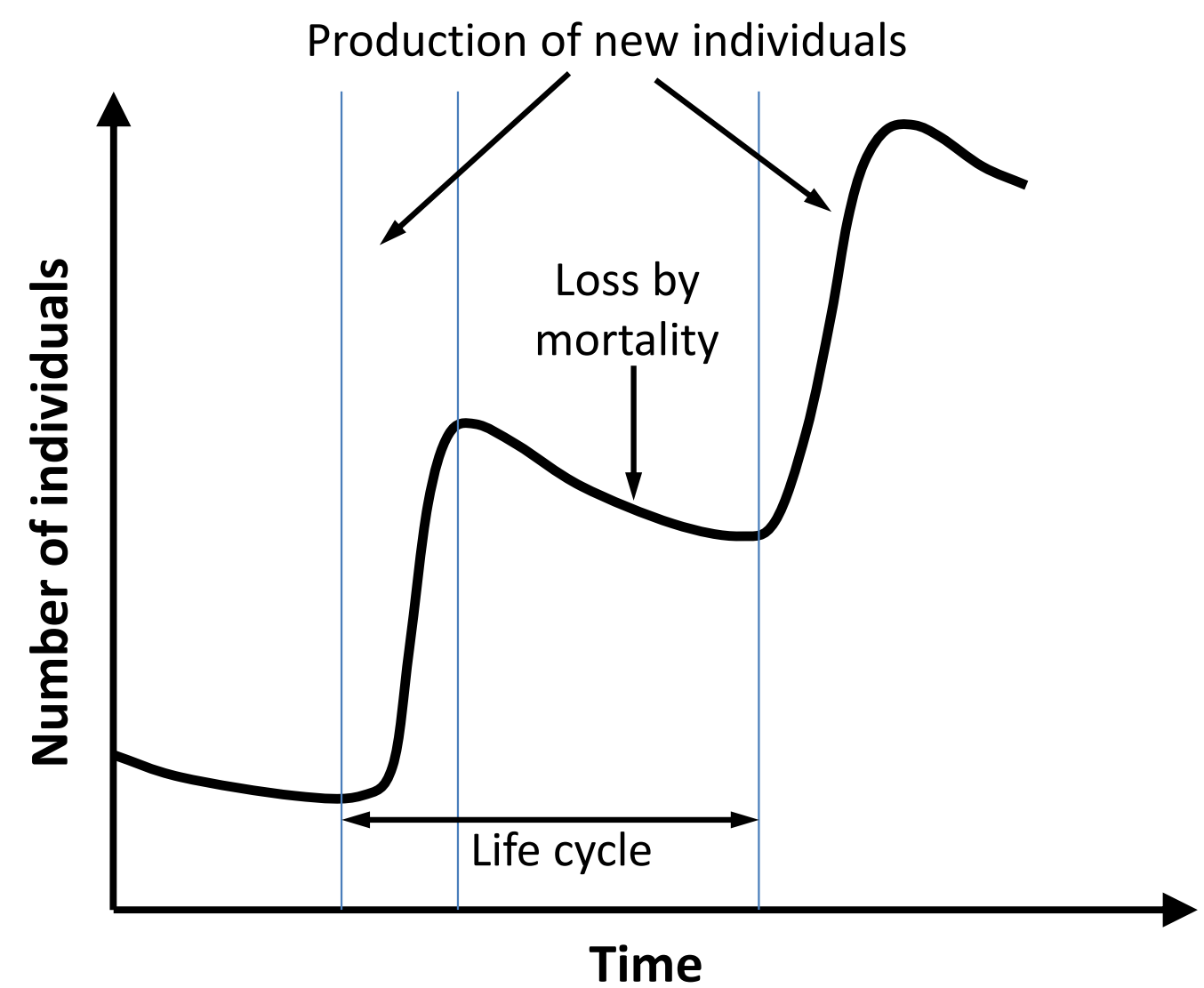

Figure 3 Total population abundance in controlled conditions. The population is initiated with newborn individuals, and decreases, first owing to mortality, until the maturation period, when a new generation is produced. At the end of the recruitment period of new individuals of second generation, the total abundance decreases, again owing to mortality. Owing to individual variability in development, loss of synchronism in population induces a broader recruitment period for the third generation. In non-limiting food conditions, recruitment of new individuals is continued after few generations. The three generations correspond to the exponential phase presented in Figure 2 (i.e., without density regulation).

To represent such patterns, it is necessary to identify different phases in the life cycle, based on age, size, developmental stages, and so on. Two types of models can be developed: 
- Structured population models (SPM) which consider the flux of individuals through different classes.

- Individual-based models (IBM) which simulate birth, growth and development through stages and death of each individual.

These models are particularly used for representing the complex life cycles of zooplankton and ichthyoplankton, but have also been useful for studying the population growth of bacteria, phytoplankton, and microzooplankton, particularly division synchrony in controlled conditions (chemostat). The major distinction between physiologically structured population models and individual-based models in a stricter sense is that individual-based models track the fate of all individuals separately over time including their states (e.g. body weight, reserve pool or cell quota) and behavior (e.g. swimming, predation), while physiologically structured population models follow population-level properties such as the density or biomass of individuals in specific classes (age or size classes, stages) using differential equations. For both model types, the physiological submodel can be represented with more or less details up to a complete budget model including a representation of structural growth and reserve pool, such as DEB models (Dynamic Energy Budget theory, Kooijman 2010)

\section{Structured population models}

A population can be structured with respect to age (age-structured population models), stage (stagestructured population models), or size or weight (size or weight-structured population models). Two types of equations systems are usually used: matrix models, which are discrete-time difference equation models, and continuous-time structured population models.

Matrix models constitute a class of population models that incorporate some degree of individual variability. Matrix models are powerful tools for analyzing, for example, the impact of life history 
characteristics on population dynamics, the influence of current population state on its growth potential, and the sensitivity of the population dynamics to quantitative changes in vital rates. Matrix models are convenient for cases where there are discrete pulses of reproduction, but not for populations with continuous reproduction. They are not suitable for studying the dynamics of populations that live in fluctuating environments. Matrix population models (Caswell, 2001) are broadly categorized into age-structured (Leslie matrix) and stage-structured (Lefkovitch matrix) models.

The Leslie matrix is the simplest type of age-structured dynamic considering discrete classes. Suppose there are $m$ age classes numbered $1,2, \ldots, m$, each covering an interval $\tau$. If $\mathrm{N}_{\mathrm{j}, \mathrm{t}}$ denotes the number of individuals in age class $j$ at time $t$ and $\mathrm{G}_{\mathrm{j}}$ denotes the fraction of the population in this age class that survive to enter age class $j+1$, then eqn [3] applies.

$\mathrm{N}_{\mathrm{j}+1, \mathrm{t}+1}=\mathrm{G}_{\mathrm{j}} \mathrm{N}_{\mathrm{j}, \mathrm{t}}$

Individuals of the first age class are produced by mature individuals from older age classes and eqn [4] applies, where $F_{j}$ is the number of age class 1 individuals produced per age class $\mathrm{i}$ individual during the time step $\tau$.

$$
\mathbf{N}_{1, \mathbf{t}+1}=\sum_{j=1}^{m} \mathbf{F}_{\mathbf{j}} \mathbf{N}_{\mathbf{j}, \mathbf{t}}
$$

The system of eqns [3] and [4] can be written in matrix form (eqn [5]).

$$
\left(\begin{array}{c}
N_{1} \\
N_{2} \\
N_{3} \\
\vdots \\
N_{m}
\end{array}\right)(t+1)=\left(\begin{array}{ccccc}
0 & F_{2} & F_{3} & \ldots & F_{m} \\
G 1 & 0 & 0 & \ldots & 0 \\
0 & G_{2} & 0 & \ldots & 0 \\
\vdots & \ddots & \ddots & \ldots & \vdots \\
0 & 0 & & G_{m-1} & 0
\end{array}\right)\left(\begin{array}{c}
N_{1} \\
N_{2} \\
N_{3} \\
\vdots \\
N_{m}
\end{array}\right)(t)
$$


The Leslie matrix can easily be modified to deal with size classes, weight classes and developmental stages as the key individual characteristics of the population. Organisms grow through a given stage or size/weight class for a given duration.

There are several variations of matrix models, differing mainly in the expression of vital rates, which can vary with time depending on external factors (e.g., temperature, food concentration, competitors, predators) or internal (e.g., density-dependent) factors. For instance, the Lefkovitch matrix is a stage-structured or size-structured model of population growth, used when demography depends on the physiological stage. The transition from one stage to another is not a time function identical for all individuals (age). It is a function depending on size or weight allowing for the inclusion of intraspecific variation in growth rates (i.e. .due to different genotypes). There are many development using matrices to represent processes of multiple populations including genetic models, dynamics of metapopulations or multispecies models (Newman et al., 2014)

The earlier type of continuous-time structured model is usually referred to as the McKendrick-von Foerster equation, and uses the age distribution on a continuous-time basis in partial differential equations. This type of model has been developed to the extent that it can be used to describe population dynamics in a fluctuating environment. In addition, it also applies to situations in which more than one physiological trait of the individuals (e.g., age, size, weight and energy reserves) have strong influences on individual reproduction and mortality. The movement of individuals through the different structural classes is followed over time. Age and weight are continuous variables whereas stage is a discrete variable.

The general equation is eqn [6], where $n$ is abundance of individuals of age $a$ and mass $m$ at time $t$.

$$
\frac{\partial \mathrm{n}(\mathrm{t}, \mathrm{a}, \mathrm{m})}{\partial \mathrm{t}}+\frac{\partial \mathrm{n}(\mathrm{t}, \mathrm{a}, \mathrm{m})}{\partial \mathrm{a}}+\frac{\partial g(\mathrm{t}, \mathrm{a}, \mathrm{m}) \mathrm{n}(\mathrm{t}, \mathrm{a}, \mathrm{m})}{\partial \mathrm{m}}=-\mu(\mathrm{t}, \mathrm{a}, \mathrm{m}) \mathrm{n}(\mathrm{t}, \mathrm{a}, \mathrm{m})
$$


The von Foerster equation describes population processes in terms of continuous age and time (agestructured models) according to eqn [7].

$$
\frac{\partial \mathrm{n}(\mathrm{a}, \mathrm{t})}{\partial \mathrm{t}}+\frac{\partial \mathrm{n}(\mathrm{a}, \mathrm{t})}{\partial \mathbf{a}}=-\mu(\mathrm{a}, \mathrm{t}) \mathrm{n}(\mathrm{a}, \mathrm{t})
$$

The equation has both an initial age structure $\varphi$ at $\mathrm{t}=0$ : (eqn [8]) and a boundary condition of egg production at $\mathrm{a}=0$ (eqn [9]).

$$
\begin{aligned}
& n(a, 0)=\varphi_{O}(a) \\
& n(0, t)=\int_{0}^{\infty} F\left(a, S_{R}\right) n(a, t) d a
\end{aligned}
$$

$\mathrm{F}$ is a fecundity function that depends on age (a) and the sex ratio of the population $\mathrm{S}_{\mathrm{R}}$. These kinds of equations are mathematically and computationally difficult to analyze, especially if the environment is not constant.

The same type of equation as [7] can be used where the age is replaced by the weight (weightstructured models, substituting $w$ to $a$ in equation7). The weight of the individual $w$ and the growth $g$ are influenced by the temperature $\mathrm{T}$, the food $\mathrm{P}$ and by the weight itself through allometric metabolic relationships, and $\mathrm{F}$ is the fecundity function which depends on weight (w) and the sex ratio of the population $S_{R}$. The numerical realization of this equation requires a representation of the continuous distribution $\mathrm{n}(w, t)$ by a set of discrete values $\mathrm{n}_{\mathrm{i}}(\mathrm{t})$ that are spaced along the weight axis at intervals $\Delta w_{\mathrm{i}}=w_{\mathrm{i}+1}-w_{\mathrm{i}}$

\section{Stage-structured population models}

Plankton populations often have continuous recruitment and are followed in the Reld by observing stage abundances over time. A large number of zooplankton population models deal with population structures in term of developmental stage, using ordinary differential equations (ODEs). 
A single ODE can be used to model each development stage or group of stages: for instance, a copepod population can be subdivided into four groups: eggs, nauplii, copepodites, and adults. The equation system is eqns [10]-[13], where $\mathrm{R}$ is recruitment, $\alpha$ is the transfer rate to next stage, and $\mu$ is the mortality rate. The system of ODEs is solved by Euler or Runge-Kutta numerical integration methods, usually with a short time step (approximately 1hour).

eggs $\quad \frac{\mathrm{dN}_{1}}{\mathrm{dt}}=\mathrm{R}-\alpha_{1} \mathrm{~N}_{1}-\mu_{1} \mathrm{~N}_{1}$

nauplii

$$
\frac{\mathrm{dN}_{2}}{\mathrm{dt}}=\alpha_{1} \mathrm{~N}_{1}-\alpha_{2} \mathrm{~N}_{2}-\mu_{2} \mathrm{~N}_{2}
$$

copepodids $\frac{\mathrm{dN}_{3}}{\mathrm{dt}}=\alpha_{2} \mathrm{~N}_{2}-\alpha_{3} \mathrm{~N}_{3}-\mu_{3} \mathrm{~N}_{3}$

adults

$$
\frac{\mathrm{dN}_{4}}{\mathrm{dt}}=\alpha_{3} \mathrm{~N}_{3}-\mu_{4} \mathrm{~N}_{4}
$$

In the model presented in Figure 4, the transfer rate of animals from stage to stage and the mortality at each stage are expressed as simple linear functions, which induce a rapidly stable stage distribution. To represent the delay of growth within a stage, more refined models consider ageclasses within each stage, or systems of delay differential equations. They have a high degree of similarity with observed cohort development in mesocosms or closed areas (Figure 5).

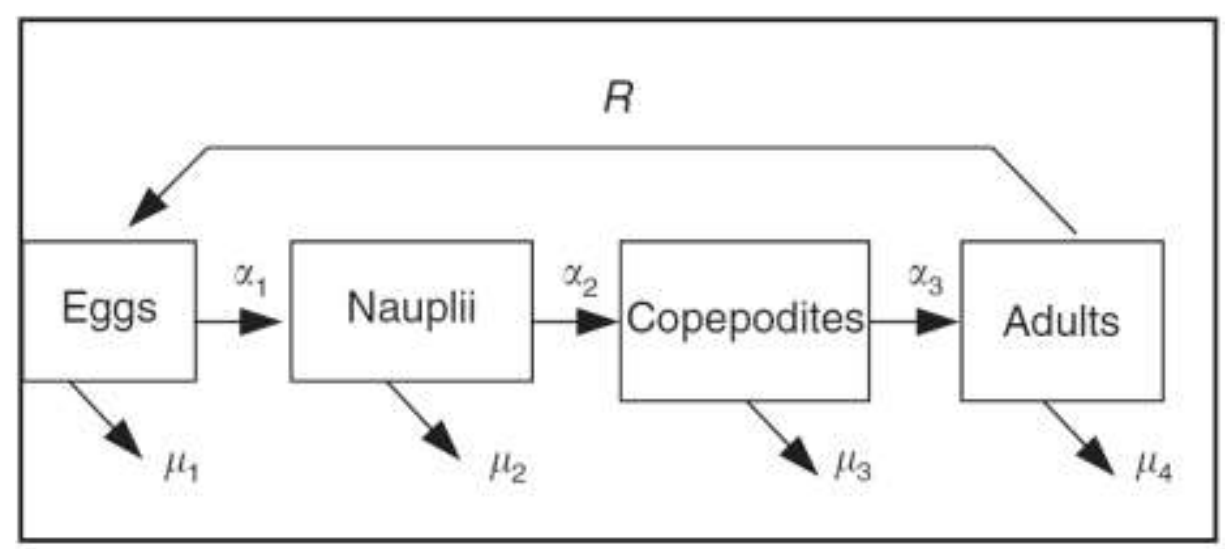

Figure 4 Schematic representation of the population dynamics mathematically represented by eqn [10]-[13]. $\alpha_{i}$ : transfer rate of stage $i$ to stage $i+1 ; \mu_{i}$ : mortality rate in stage $\mathrm{i} ; \mathrm{R}$ : recruitment: number of eggs produced by females per day. 

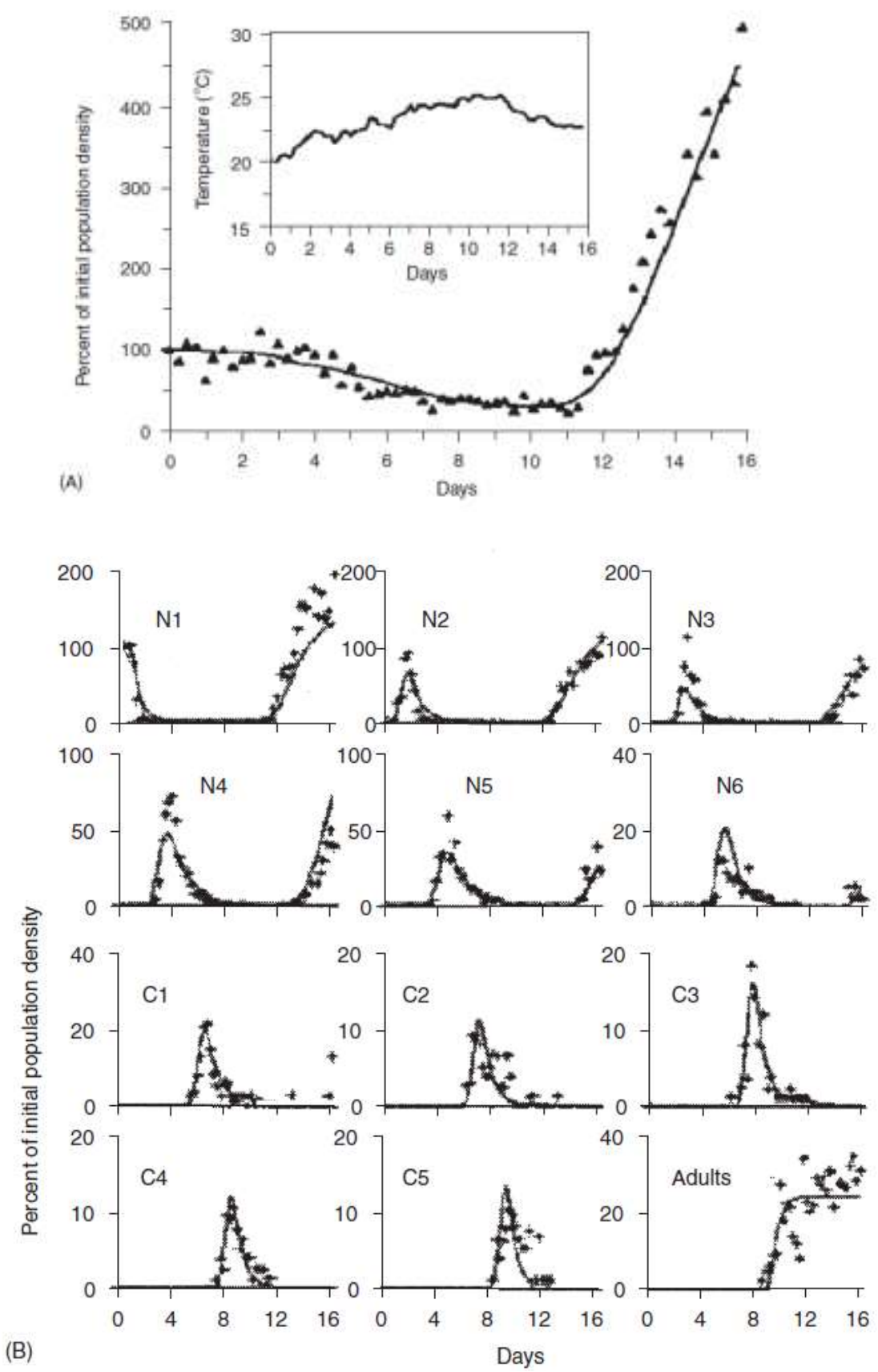

Figure 5 Simulation of the cohort development of the copepod Euterpina acutifrons in mesocosms with a structured stage and age-within-stage model. (A) Total population during development, with variable temperature and constant food supply (points = experimental data; line $=$ simulation). The initial density decreases owing to mortality and then increases to new born individuals (as the first part in Figure 3). (B) Naupliar stages (N1 to N6), copepodite stages (C1 to C5), and adults during development (points $=$ experimental data; line $=$ simulation). The simulation starts with similar N1 of same age. Reproduced from Carlotti F and Sciandra A, 1989. Population dynamics model of Euterpina acutifrons (Copepoda: Harpacticoida) coupling individual growth and larval development. Mar. Ecol. Prog. Ser., 56, 3, 225-242. 


\section{Individual-based models of a population}

Because transport of planktonic organisms is largely controlled by oceanic currents, numerous IBM of planktonic populations, or of planktonic larval forms of nektonic and benthic populations, have been developed and coupled to hydrodynamic models of varying degrees of three-dimensional and temporal complexity. IBMs have been applied for phytoplankton and zooplankton species, meroplanktonic larvae and early life-history of fish populations. Individual-based modeling approach is well established for metazoans where organisms are more functionally complex, but are increasingly being applied to unicellular organisms when growth kinetics are formulated as a function of internal properties. Object-oriented programming (OOP) and cellular automata are techniques that have been applied to IBMs recently.

IBMs do not make the same averaging assumption as SPMs, which can be important when model equations are nonlinear and heterogeneity exists, and they are more suitable for simulating complex and adaptive behavior. IBMs treat populations as collections of individuals, with explicit rules governing individual biology and interactions with the environment. Each biological component can change as a function of the others. Each individual is represented by a set of variables that store its $i$ state (e.g. age, size, weight, nutrient or reserve pool, etc.). These variables may be grouped together in some data structure that represents a single individual, or they may be collected into arrays (an array of all the ages of the individuals, an array of all the sizes of the individuals, etc.), in which case an individual is an index number in the set of arrays. The i-state of an individual changes as a function of the current i-state, the interactions with other individuals, and the state of the local environment. The local environment can include prey and predator organisms that do not warrant explicit representation as individuals in the model. Population-level phenomena (e.g., temporal or spatial dynamics) or vital rates can then be inferred directly from the contributions of individuals in the ensemble. 
The model starts with an initial population and the basic environment, then monitors the changes of each individual. At any time t, the $i$-state of individual $\mathrm{j}$ changes as eqn [14].

$$
X_{i, j}(t)=X_{i, j}(t-d t)+f\left(X_{1, j}(t-d t), \ldots ., X_{i, j}(t-d t)\right.
$$

$X_{i, j}(t)$ is the value of the $i$-state of individual $j$, and $f$ is the process modifying $X_{i, j}$, as a function of the values of different i-states of the organism, and external parameters such as the temperature. When the fate of all individuals during the time-step $d t$ has been calculated, the changes to the environment under the effects of individuals can be updated. Any stochastic process can be added to eqn [14].

This type of model can add a lot of detail in the representation of physiological functions. Individual growth can be calculated as assimilation less metabolic loss, and the inter-individual variation in physiology can be represented by adding stochastic processes or parameters describing the characteristics of each individual (growth and development parameters, mortality coefficient, and parameters connected with reproduction). The end results are unique life histories, which when considered as a whole give rise to growth/size distributions that provide a measure of the state of the population Individual traits and population structure and dynamics emerge from the properties of metabolic organization and the indirect interactions of individuals via competition for food.

\section{Calibration of parameters}

Parameterization of a model can range from very simplistic to extremely complex depending upon the amount of information known about the population under consideration. Bioenergetic processes (ingestion, egestion, excretion, respiration and egg production) are usually modeled from experimental results, whereas biometrics (size, weight, ...) and demographic (development rate, mortality rate, ) parameters are estimated by combining data from life table collected in the field or from laboratory studies. 
Calibration task involves systematic adjustment of model parameter estimates so that model outputs more accurately reflect observed data.. To solve for the unknown parameters, new techniques have been developed such as inverse methods and data assimilation by fitting simulations to data. The use of allometric relationships of physiological rates to weight or applying conceptual frame such as the DEB theory where underlying physiological processes based on first principles (e.g. massenergy conservation laws) allows reducing the number of parameters

\section{SPATIAL DISTRIBUTION OF SINGLE PLANKTON POPULATIONS}

An important development in plankton population modeling is to make full use of the increased power of computers to simulate the dynamics of plankton (communities or populations) in sitespecific situations by coupling biological and transport models, giving high degrees of realism for interpreting plankton population growth, transport, spatial distribution, dispersion, and patchiness.

These models fall into two basic categories to represent spatial plankton dynamics (1) Eulerian or grid-based models which follow the evolution of some quantity (abundance or biomass of structured population models) at discrete, fixed physical locations using advection-diffusion-reaction equations, and (2) Lagrangian biological models which follow individual organisms or groups of organisms moving through space as they are advected by the ambient currents with the biology of each organism described by an IBM module. Continuous development in physical -biological coupled modeling technique for both types of models have opened opportunities to study the variability of the seasonal population cycles under different scenarios of environmental forcing for different regions of the ocean (Ji et al., 2010)

\section{Spatial plankton dynamics with advection-diffusion-reaction equations}

Eulerian or grid-based models are used to simulate the distribution of variables that behave like tracers (e.g. plankton concentrations). The state of a variable in fixed space volumes (i.e. boxes or 
grid cells) is represented by a single average value and the dynamics in the field domain of the grid model depends on the biological changes within each box and exchanges between boxes. Equation [15] is the general physical-biological model equation used to describe the interaction between physical mixing and biology.

$\frac{\partial \mathrm{C}}{\partial \mathrm{t}}+\nabla \cdot\left(v_{\mathrm{a}} \mathrm{C}\right)-\nabla \cdot(\mathrm{K} \nabla \mathrm{C})="$ biological terms"

$C(x, y, z, t$,$) is the concentration of the biological variable at position x, y, z$ at time $t$ (expressed as numbers of organisms or biomass of organisms per unit volume) which could be either a planktonic functional group (e.g. zooplankton), or a species or a size class (in which case the number of equations would equal the number of size classes). . $v_{a}\left(u_{a}, v_{a}, w_{a}\right)$ represents the advective fluid velocities in $x, y, z$ directions. $K_{x}, K_{y}, K_{z}$ are diffusivities in $x, y, z$ directions. $\nabla=(\partial / \partial \mathrm{x}, \partial / \partial \mathrm{y}, \partial / \partial \mathrm{z})$ is the Laplacian operator.

On the left hand side of eqn [15], the first term is the local change of $\mathrm{C}$, the second term is advection caused by water currents, and the third term is the diffusion or redistribution term. The right hand side of eqn [15] has the biological terms that represent the sources and sinks of the biological variable at position $x, y, z$ as a function of time. The biological terms may include a velocity component (swimming of organisms, migrations, sinking, ...), and the complexity of the biological representation can vary from the dispersion of one (the concentration of a cohort) to detailed population dynamics.

Physical-biological models of various levels of sophistication have been developed recently for different regions of the ocean. Biological models can be configured as compartmental ecosystem models in an upper ocean mixed-layer, where phyto-, microzoo- and meso-zooplankton are represented by one variable. In extended case, the model takes into account several size classes of phyto-, microzoo- and zooplankton. Recent model developments consider concurrent species with variable functional traits (e.g. Darwin model, Follows et al. 2007). Studies of plankton population distribution in heterogeneous marine landscape where plankton may be aggregated in dynamical 
mesoscale structures (e.g. upwelling- and downwelling- regions, Langmuir circulations, eddies) can be undertaken with population described by equations of the McKendrick-von Foester type coupled with 2D or 3D fine-grid hydrodynamical models (Runge et al., 2005).

A classic example by Wroblewski in 1982 is the stage-structured population model of Calanus marshallae, a copepod species, embedded in a circulation system simulating the upwelling off the Oregon coast (Figure 6). Simulations of the dynamics focused on the interaction between diel vertical migration and offshore surface transport. The zonal distribution of the life stage categories $C_{i}$ of $C$. marshallae over the Oregon continental shelf was modeled by the two-dimensional $(x, z, t)$ equation [16], where the vertical swimming speed of the $i$ th stage $w_{b i}$ follows a sinusoidal function of time: $w_{b i}=w_{s i} \sin (2 \pi t)$, with $w_{s i}$ the maximum migration speed of the stage.

$$
\begin{aligned}
& \frac{\partial \mathrm{C}_{\mathrm{i}}(\mathrm{x}, \mathrm{z}, \mathrm{t})}{\partial \mathrm{t}}-\frac{\partial\left[\mathrm{u}_{\mathrm{a}}(\mathrm{x}, \mathrm{z}, \mathrm{t}) \mathrm{C}_{\mathrm{i}}(\mathrm{x}, \mathrm{z}, \mathrm{t})\right]}{\partial \mathrm{x}}-\frac{\partial\left[\mathrm{w}_{\mathrm{a}}(\mathrm{x}, \mathrm{z}, \mathrm{t}) \mathrm{C}_{\mathrm{i}}(\mathrm{x}, \mathrm{z}, \mathrm{t})\right]}{\partial \mathrm{z}} \\
& -\frac{\partial}{\partial \mathrm{x}}\left[\mathrm{K}(\mathrm{x}, \mathrm{t}) \frac{\partial \mathrm{C}_{\mathrm{i}}(\mathrm{x}, \mathrm{z}, \mathrm{t})}{\partial \mathrm{x}}\right]-\frac{\partial}{\partial \mathrm{z}}\left[\mathrm{K}(\mathrm{z}, \mathrm{t}) \frac{\partial \mathrm{C}_{\mathrm{i}}(\mathrm{x}, \mathrm{z}, \mathrm{t})}{\partial \mathrm{z}}\right] \\
& =\text { populationdynamics }+\frac{\partial\left[\mathrm{w}_{\mathrm{bi}}(\mathrm{x}, \mathrm{z}, \mathrm{t}) \mathrm{C}_{\mathrm{i}}(\mathrm{x}, \mathrm{z}, \mathrm{t})\right]}{\partial \mathrm{z}}
\end{aligned}
$$

The population dynamics model was presented in equations [10] to [13]. The upwelling zone extended $50 \mathrm{~km}$ from the coast down to a depth of $50 \mathrm{~m}$, and was divided into a grid with spacing $2.5 \mathrm{~m}$ in depth and $1 \mathrm{~km}$ in the horizontal. The author used a finite difference scheme with a time step of 1 hour, which fell within the bounds for computational stability. 


\section{Distance offshore $(\mathrm{km})$}

(A)

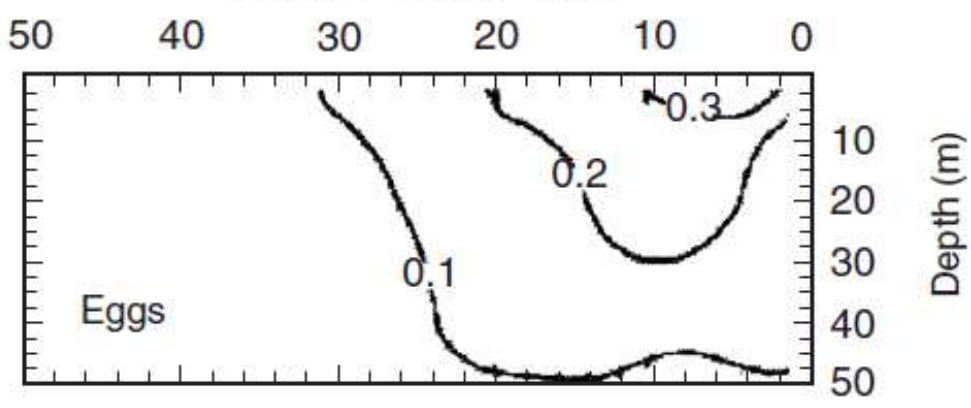

(B)

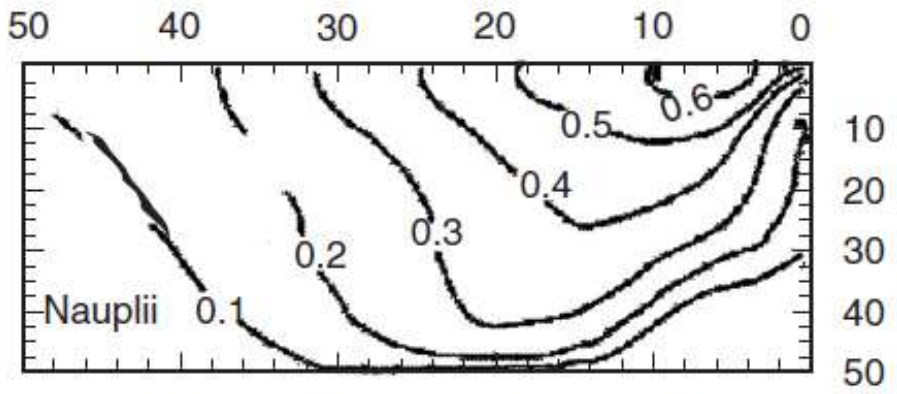

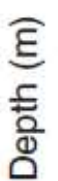

(C)

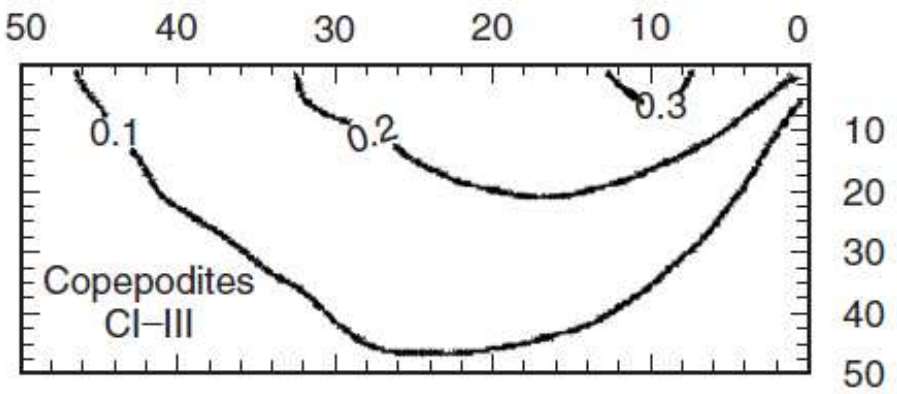

Figure 6 Model of Calanus marshallae in the Oregon upwelling zone. The figure shows the simulated zonal distribution of (A) eggs, (B) nauplii, and (C) early copepodites at noon on 15 August. Concentrations of each stage are expressed as a fraction of the total population (all stages $\left.\mathrm{m}^{-3}\right)$. Reproduced from Wroblewski JS, 1982. Interaction of currents and vertical migration in maintaining Calanus marshallae in the Oregon upwelling zone-a simulation. Deep Sea Research 29: 665-686.

\section{Coupling IBMs and spatially explicit models}

This approach uses simulated currents from sophisticated 3D hydrodynamic models driving Lagrangian models of particle trajectories to examine dispersion processes. IBMs of planktonic species are associated with sparsely distributed particles, each representing an individual planktonic 
organism or a group of identical individuals, which are forced with output from an Eulerian biophysical model (i.e. one-way coupling) of varying degrees of 3D and temporal complexity. The approach is relatively straightforward and is a first step in formulating spatially-explicit individual based models. Given a "properly resolved" flow field, particle trajectories are computed (generally with standard Runge-Kutta integration methods of the velocity field). Specifically, hydrodynamic models provide the velocity vector $\mathbf{v}=(u, v, w)$ as a function of location $\mathbf{x}=(x, y, z)$ and time $\mathrm{t}$ and the particle trajectories are obtained from the integration of eqn [17].

$$
\mathrm{d} \mathbf{x} / \mathrm{dt}=\mathbf{v}(\mathrm{x}, \mathrm{y}, \mathrm{z}, \mathrm{t})
$$

The simplest model of dispersion is a random walk model in which individuals move along a line from the same starting position. These trajectories could be modified by turbulent dispersion as described in the section below. Once the larval/particle position is known, additional local physical variables can be estimated along the particle's path: e.g., temperature, turbulence, light, etc., and input to the IBM. The physical quantities are then included in biological (physiological or behavioural) formulations of IBMs (see below).

By coupling an IBM population of key species with its environmental forcing (food, temperature) provided by a coupled physical- biogeochemical model, the simulations allow to evaluate the influence of environmental variability of the bio-physical environment (flow, temperature, turbulence, light, and trophic resources) on dispersal, growth and mortality of planktonic life stages and to explain how patchy habitats may influence the distribution of individuals within a population and recruitment in a given area. Simulations considering trajectories of plankton as passive particles are usually a first step before considering any active swimming capability of planktonic organisms either using a prescribed vertical migration strategy, or integrating a vertical motion (sinking or swimming) which could depend on animal stage development and/or its physiological state. The simulated plankton distributions from these models tend to compare better with observed 
distributions than models that use passive particles. Sensitivity studies show that behaviour is an important factor in determining larval transport and/or retention.

The coupling of IBMs of zooplankton and fish populations and 3D circulation models is still a recent field of study (Figure7). Available on-line user-friendly design of lagrangian tools such as Ichthyop (http://www.ichthyop.org; Lett et al., 2008) allows to simulate zoo- and ichthyoplankton dynamics in any oceanic region by incorporating the most important processes involved in plankton or fish early life, and to force the IBM module by input time series of velocity, temperature and salinity fields archived from ROMS.

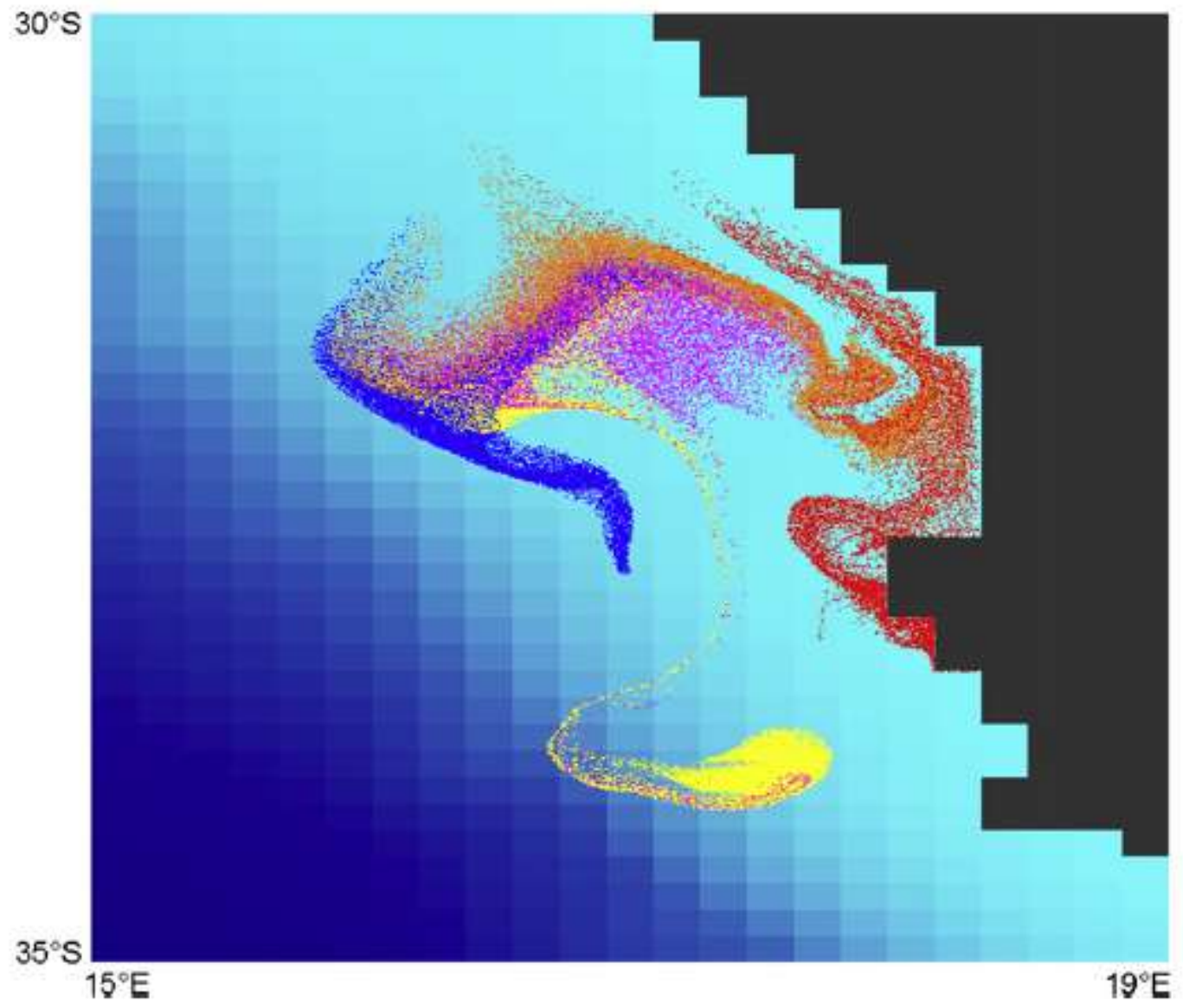

Figure 7: Simulated IBM of fish eggs and larvae dispersal pattern displayed on a longitude latitude plot with the ichthyop tool (http://www.ichthyop.org). Reproduced from Lett et al. (2008) A Lagrangian tool for modelling ichthyoplankton dynamics.. Environmental Modelling and Software, Elsevier, 23 (9), 1210-1214. 
Generally, models that describe the spatial heterogeneity of the habitat have been designed to answer questions about the spatial and temporal distribution of a population, particularly about connectivity of metapopulations between habitats (e.g. Cowen et al 2009). They allow us to explore the potential effects of habitat alteration on these populations.

\section{Modeling behavioral mechanisms, aggregation and schooling, patches}

Different types of models have been built, some of them focusing on the structure and shape of aggregations depending on internal and external physical forces, others dealing with the benefits for individuals of living in groups with regard to feeding (foraging models) and to predation. The Lagrangian approach can take into account the behavior of individual organisms, and the effects of the physical environment upon them. Although Eulerian approaches are mathematically tractable, the methods do not explicitly address the density dependence of aggregating individual behavior within a patch.

Dynamic optimization allows descriptions of the internal state of individuals, which may lead to both variable and fluctuating motivations among individuals over short time periods

\section{INTERACTIONS BETWEEN POPULATIONS}

\section{Models with plankton populations in interactions}

Interactions between populations by competition for shared resources and by predation are among the most important factors driving plankton community dynamics. Interaction between marine species have been rarely treated by stage-resolved population models, although structured population models as well as IBM models can represent interactions between species like predation, parasitism or even cannibalism.

Simple models of two species interactions take the form of eqns [18] and [19] (The so-called “Lotka-Volterra equations" or predator-prey equations): 


$$
\begin{aligned}
& \frac{\mathrm{dN}}{\mathrm{dt}}=\mathrm{r}_{1} \mathrm{~N}_{1}-\mathrm{k}_{1} \mathrm{~N}_{1} \mathrm{~N}_{2} \\
& \frac{\mathrm{dN} \mathrm{N}_{2}}{\mathrm{dt}}=\mathrm{r}_{2} \mathrm{~N}_{2}-\mathrm{k}_{2} \mathrm{~N}_{1} \mathrm{~N}_{2}
\end{aligned}
$$

Different extensions of the second term on the right part of the equation can be used. Such equations have been used in many situations of predator-prey interaction (e.g. bacteria-phage, carnivorous zooplankton - prey species) or between species of the same trophic level in competition for resources As an example, Gaedke and Ebenhöh presented in 1991 (reference in Carlotti et al. 2000) a study on the interaction between two estuarine species of copepods Acartia tonsa and Eurytemora affinis. They first used a simple model based on eqns [18] and [19] representing two bulk populations in interaction, and secund two-stage-structured population models with similar eqns to [10]-[13]) including crossed interactions between stages of the two species. Only the second model resulted in stable coexistence between the two species due to the predation on larval stages of both species by Acartia tonsa.

The original "kill the winner" (KtW) model (see references in Lindeman et al. 2017) explaining the continual succession of different planktonic populations in competition for resources is formulated as deterministic Lotka-Volterra-type equations for the species biomass concentrations. Nowadays, KtW models have been applied to planktonic communities consisting of viruses; prokaryotes, phytoplankton and zooplankton protozoans and metazoans).

\section{Food webs with population models}

In the last decades, there has been an increasing use of ecosystem models including representation of multiple phytoplankton and zooplankton populations in interaction (Plankton Functional Type models) and coupled to physical models to study the effect of biodiversity on ecosystem functioning 
(e.g. the Darwin model, Follows et al 2007 ). The different species represent differing degrees of functional diversity defined by species' functional traits. Phytoplankton species compete for nutrient resources and represent resources for zooplankton species which additionally exert cannibalism, and so on for zooplankton predators. The planktonic community structure emerges from a range of possibilities determined by processes of dispersal, competition for resources, predation and physiological characteristics.

Although the amount of energy transferred through the pelagic food chain from phytoplankton to higher trophic levels can be represented by food web models taking into account bulk species biomass (Ecopath), size-based food-web approach is preferred by assuming species- and size-based interactions which result from the dominance of small primary producers, size-based predation and ontogenetic increases in the trophic levels of many consumers. The output of these models is that the community structure is an emergent property of the ecosystem functioning itself. 
See also: Biogeochemical Data Assimilation (xxx). Carbon Cycle (xxx). Fish Larvae (2xxx 6). Fish Predation and Mortality (xxx). Fish Vertical Migration (xxx). Gelatinous Zooplankton (xxx). Krill (xxx). Lagrangian Biological Models (xxx). Large Marine Ecosystems (xxx). Marine Mesocosms (xxx). Microbial Loops (xxx). Models of Small-scale Patchiness (xxx). Network Analysis of Food Webs (xxx). Nitrogen Cycle (xxx). Ocean Gyre Ecosystems (xxx). Patch Dynamics (xxx).

Phosphorous Cycle (xxx). Polar Ecosystems (xxx). Small-scale Physical Processes and Plankton Biology (xxx). Upwelling Ecosystems (xxx). Zooplankton (xxx). 


\section{References}

Brun P, Kiørboe T, Licandro P, Payne M (2016) The Predictive Skill of Species Distribution Models for Plankton in a Changing Climate John Wiley \& Sons Limited,

Carlotti F, Giske J and Werner F (2000) Modelling zooplankton dynamics. In: Harris RP, Wiebe P, Lenz J, Skjoldal HR and Huntley M (eds) Zooplankton Methodology Manual, pp. 571-667. New York: Academic Press.

Caswell H (2001) Matrix Population Models: Construction, Analysis, and Interpretation , 2nd ed.. Sunderland, MA: Sinauer Associates

Cowen R, Gawarkiewicz G, Pineda J, Thorrold S and Werner, F (2007) Special Issue: Marine Population Connectivity. Oceanography. 20 (3).

Fennel W and Neumann T (2014) Introduction to the Modelling of Marine Ecosystems. Elsevier Amsterdam: Oceanography Series Volume 72 2nd Edition

Follows MJS, Dutkiewicz S., Grant S and Chisholm SW (2007) Emergent biogeography of microbial communities in a model ocean, Science, 315, 1843-1846.

De Angelis DL and Grimm V. (2014). Individual-Based Models in Ecology after Four Decades. F1000Prime Reports $6: 39$. 
Ji R, Edwards M, Mackas DL, Runge JA and Thomas A.C. (2010) Marine plankton phenology and life history in a changing climate: current research and future directions. Journal of Plankton Research 32, 1355-1368.

Kooijman S (2010) Dynamic Energy Budget theory for metabolic organization . Cambridge University Press

Lewis MA, Maini PK, Petrovskii SV (2013) Dispersal, individual movement and spatial ecology : a mathematical perspective. Lecture notes in mathematics 2071 Springer-Verlag Berlin Heidelberg

Lindemann C, Aksnes DL, Flynn KJ and Menden-Deuer S (2017) Modeling the PlanktonEnhancing the Integration of Biological Knowledge and Mechanistic Understanding. Front. Mar. Sci. 4:358 (special issue)

Newman, K., Buckland, S.T., Morgan, B., King, R., Borchers, D.L., Cole, D., Besbeas, P., Gimenez, O., Thomas, L. (2014) Modelling population dynamics: model formulation, fitting and assessment using state-space methods. Methods in statistical ecology. New York: Springer.

Rose KA, Allen JI, Artioli Y, Barange M, Blackford J, Carlotti F, Cropp R, Daewel U, Edwards K, Flynn K, Hill SL, Hille Ris Lambers J, Huse G, Mackinson S, Megrey B, Moll A, Rivkin R, Salihoglu B, Schrum C, Shannon L, Shin Y-J, Smith SL, Smith C, Solidoro C, St. John M and Zhou M (2010). End to end models for the analysis of marine ecosystems: challenges, issues, and next steps. Mar. Coast. Fish. 2: 115-130 
Runge J A, Franks PJS, Gentleman WC, Megrey B.A., Rose K.A., Werner, F.E. and Zakardjian B. (2005) Diagnosis and prediction of variability in secondary production and fish recruitment processes: developments in physical-biological modelling. In Robinson A. R. and Brink K. (eds), The Global Coastal Ocean: Multi-Scale Interdisciplinary Processes, The Sea, Vol. 13. Harvard University Press, Cambridge, MA, pp. 413-473.

Soetaert K. and Herman PMJ (2009): A Practical Guide to Ecological Modelling Using R as a Simulation Platform. Springer Verlag. 